\title{
PRESCRIBING WRITING ERRORS IN FAMILY HEALTH UNITS IN ZAGAZIG CITY: A COMPARATIVE STUDY BETWEEN ACCREDITED AND NON-ACCREDITED HEALTH UNITS.
}

Shereen M. Bahgat*, Marwa M.Saed, MD Nagwa E. Sobhy, MD and Mohamed A.Fouda*, MD

Family Medicine Department, Kasr El-ini, Cairo University and Community Medicine Department*, Faculty of Medicine, Zagazig University

Objective:In this study, the main objective was to determine prescription errors in family health units in Zagazig city accredited versus non accredited and its relation to the organizational climate.

Patients \&Method:10 accredited family health units were included in the study. Comparing the prescription of these unit to the standard of the ministry of health and WHO.

Results: It was found that the frequency of errors in accredited unit were fewer. The accreditation affects both the frequency of errors and the organizational climate, as the accredited units showed fewer errors and higher organizational climate score than the non-accredited units. There was a negative correlation between the organizational climate and the frequency of missing errors.

Conclusions: Prescribers could be helped by designing systems to reduce the risk of these errors like that of a continuous education training programs and use of computerized prescription. The prescriptions need more effort and support from all the related to it; from the doctors, senior managers to the accreditation committee and ministry of health.

Key words: prescribing, errors, family unit, organizational climate.

\section{INTRODUCTION}

$\mathbf{P}$ rescriptions errors are a serious public health problem and have received a great deal of attention in recent years. Their effects can range from small injuries to the patient's death and these errors can occur at any stage of the medication system. For these reasons, various studies have aimed to analyze medication systems, detect the most frequent problems, remove the focus from human caused errors and, above all, improve patient safety ${ }^{(1)}$.

Prescription deficiencies formed a large proportion of errors identified in prescription screening ${ }^{(2)}$. This is mainly due to the attitude of some prescribers who are always in a hurry and hence unwilling to spend a little more time in writing clear and complete prescriptions. However, the extra time spent on the prescription will help to ensure that the patient receives the treatment that is intended by the prescriber. Errors in prescribing may be classified into two main types: errors of omission (incomplete prescription) and errors of commission (incorrect information) ${ }^{(3)}$.

Prescribing errors involving decision making include a wrong choice for the patient (due to allergies, interactions between two drugs, presence of liver or renal failure, wrong molecule, dose or route of administration, etc.).Prescription errors in prescription writing (omission), instead, involve illegibility, ambiguous abbreviations, lack of an important piece of information such as date of prescription, dose, route, frequency of administration, etc ${ }^{(3)}$.

World Health Organization (WHO) declared in 2008 that no information is yet available for developing countries and those with economies in transition; however, as access to medications improves, it is reasonable to assume that adverse drug events will also become an important source of morbidity, mortality and financial liability in those countries, which already have resource-strapped health systems ${ }^{(4)}$.

This study aims to assess the prescription writing quality in family medicine unites in Zagazig city, which helps to assess prescribing conformity to the standard regarding the omission.

\section{Technical Design:}

\section{SUBJECTS AND METHODS}

The study was conducted in accredited and non-accredited family health units in Zagazig city. There is 45 family health unit in Zagazig city; 11 accredited and 34 non-accredited. In the pilot study 1 accredited unit versus 1 non-accredited unit had been included. So the rest of the units had been included in the study.

\section{Sample technique and size:}

Twenty Family Health unites in Zagazig city (10 accredited versus 10 non-accredited). All doctors works in these units were included in the study, who there were 54 doctors with approximately 30 prescription for every doctor; the pharmacists were included only in gap analysis 
questionnaire and the employees at the front desk were included in the educational intervention as they play role in writing name, age, address and sex of the patients.

\section{Inclusion criteria:}

- Physicians working for more than 6 months in these units.

- Pharmacists in the units.

- Employee in the front desk.

Tools of data collection:

- Questionnaires for relevant personal data (name, age, Education level, Work experience, work satisfaction, knowing of presence of standard and the source if it is official or not, caring to write prescription according it and to any percent).

- Standard for prescription writing (patient name, age, sex, address, date, diagnosis in Arabic, generic name of the drug clearly in Arabic, concentration of the drug, dose, route of administration, duration of drug intake, follow up and signature of the prescriber).

- Questionnaire for organizational climate including 10 categories and every category contain 5 questions (organizational design, individual job characteristics, co-work relations, culture/work environment, senior management, direct supervisor, work process, communications, technology and customer satisfaction).The questionnaire was obtained from www.plusdelta.net., and little change was done to fit the work I the health units.

All the questionnaires were proved to be reliable via Alpha Croubach test with value 0.75.

\section{$>$ Preparatory phase:}

The researcher reviewed the past and current related literature, textbooks, journals and internet services. The personal and environmental working condition questionnaire was designed and the WHO and MOHP guidelines in prescription writing were reviewed.

\section{Pilot study:}

It was done on $10 \%$ of the sample check the validity and feasibility of the practical work. Pilot test was undertaken before finalizing all the sheets. Before the start of the study, the sheets were pretested and insignificant modifications were done. The sheets were pre-tested several times to ensure that the wording, format, length, and sequencing of questions were appropriate. During each successive pre-test, feedback was obtained to help refine quality of the measures. The unit of the pilot study was not included in the study.

\section{$>$ Data analysis, interpretation \& writing}

The collected data was tabulated and summarized, then analyzed using the appropriate descriptive statistical and analytical tests in order to check the research effectiveness.

\section{Administrative Design and Ethical Aspect}

The ethical approval was achieved by ethical committee in the department, the faculty, MOHPand informed written consent by the doctors.

\section{Data Management}

By SPSS program version 12 using the appropriated descriptive statistical and analytical tests to measure the statistical difference between the pre and post intervention checklist and the difference of degree of education on the service quality.

The organizational climate score calculation:

Every category in the organizational climate have a total score 25 , then the answers was summed then divided from 25 to get the percent. If this percent $<60$, so it is inadequate. If $>60$, so it is adequate. 
RESULTS

Table (1): Physicians' qualification, and experience in the accredited and non-accredited units

\begin{tabular}{|c|c|c|c|c|c|c|}
\hline & \multicolumn{4}{|c|}{ Center } & \multirow{3}{*}{$\underset{\text { test }}{\chi^{2}}$} & \multirow{3}{*}{ p-value } \\
\hline & \multicolumn{2}{|c|}{ Accredited } & \multicolumn{2}{|c|}{ Non-accredited } & & \\
\hline & No. & $\%$ & No. & $\%$ & & \\
\hline \multicolumn{7}{|l|}{ Qualification: } \\
\hline Bachelor & 9 & 33.3 & 9 & 33.3 & & \\
\hline Master & 6 & 22.2 & 8 & 29.6 & 3.34 & 0.34 \\
\hline Egyptian family medicine fellowship & 3 & 11.1 & 0 & 0.0 & & \\
\hline Diploma & 9 & 33.3 & 10 & 37.0 & & \\
\hline \multicolumn{7}{|l|}{ Experience years: } \\
\hline $1-$ & 14 & 51.9 & 11 & 40.7 & & \\
\hline $5-$ & 10 & 37.0 & 11 & 40.7 & 0.86 & 0.35 \\
\hline $10+$ & 3 & 11.1 & 5 & 18.5 & & \\
\hline
\end{tabular}

Chi square shows no significant difference in all items $(\mathrm{P}>0.05)$.

Table (2): Frequency of missing errors in prescribing in the accredited and non-accredited units For every doctors $\approx 30$ prescriptions

\begin{tabular}{|c|c|c|c|c|c|c|}
\hline \multirow{3}{*}{ Missing errors in: } & \multicolumn{4}{|c|}{ Center } & \multirow{3}{*}{$\begin{array}{c}\chi^{2} \\
\text { test }\end{array}$} & \multirow{3}{*}{ p-value } \\
\hline & \multicolumn{2}{|c|}{ Accredited } & \multicolumn{2}{|c|}{$\begin{array}{c}\text { Non- } \\
\text { accredited } \\
\end{array}$} & & \\
\hline & No. & $\%$ & No. & $\%$ & & \\
\hline Patient name & 0 & 0.0 & 0 & 0.0 & 0.00 & 1.00 \\
\hline Patient age & 0 & 0.0 & 0 & 0.0 & 0.00 & 1.00 \\
\hline Patient sex & 24 & 88.9 & 27 & 100.0 & Fisher & 0.24 \\
\hline Patient address & 24 & 88.9 & 24 & 88.9 & Fisher & 1.00 \\
\hline Date & 0 & 0.0 & 0 & 0.0 & 0.00 & 1.00 \\
\hline Diagnosis in Arabic & 11 & 40.7 & 13 & 48.1 & 0.30 & 0.58 \\
\hline Scientific name of drug clearly in Arabic & 14 & 51.9 & 20 & 74.1 & 2.86 & 0.09 \\
\hline Concentration of the drug & 11 & 40.7 & 22 & 81.5 & 9.43 & $0.002 *$ \\
\hline Dose & 10 & 37.0 & 24 & 88.9 & 15.56 & $<0.001^{*}$ \\
\hline Route of administration & 3 & 11.1 & 14 & 51.9 & 10.39 & $0.001 *$ \\
\hline Duration of drug intake & 15 & 55.6 & 22 & 81.5 & 4.21 & $0.04 *$ \\
\hline Follow up & 27 & 100.0 & 27 & 100.0 & 0.00 & 1.00 \\
\hline Signature of the prescriber & 17 & 63.0 & 27 & 88.9 & 4.96 & $0.03 *$ \\
\hline
\end{tabular}

(*) Statistically significant at $\mathrm{p}<0.05$

Chi square shows highly significant difference in items of dose of the drug and route of its administration, where there is significant difference in concentration of the drug, duration of its take and the signature of the prescriber. 
Table (3): Total organizational climate adequacy as reported by physicians in the accredited and non-accredited units

\begin{tabular}{|c|c|c|c|c|c|c|}
\hline \multirow{3}{*}{$\begin{array}{c}\text { Adequate } \\
\text { Organizational climate } \\
\text { Domains }\end{array}$} & \multicolumn{4}{|c|}{ Center } & \multirow{3}{*}{$\begin{array}{c}\chi^{2} \\
\text { test }\end{array}$} & \multirow{3}{*}{$\mathrm{p}$-value } \\
\hline & \multicolumn{2}{|c|}{ Accredited } & \multicolumn{2}{|c|}{ Non-accredited } & & \\
\hline & No. & $\%$ & No. & $\%$ & & \\
\hline Organizational design & 27 & 100 & 15 & 55.6 & 15.43 & $<0.001^{*}$ \\
\hline Individual job characteristics & 11 & 40.7 & 8 & 29.6 & 0.73 & 0.39 \\
\hline Co-work relations & 25 & 92.6 & 4 & 14.8 & 32.85 & $<0.001^{*}$ \\
\hline Culture/work environment & 24 & 88.9 & 3 & 11.1 & 32.67 & $<0.001^{*}$ \\
\hline Senior management & 18 & 66.7 & 21 & 77.8 & 0.83 & 0.36 \\
\hline Direct supervisor & 13 & 48.1 & 8 & 29.6 & 1.95 & 0.16 \\
\hline Work process & 25 & 92.6 & 17 & 63.0 & 6.86 & $0.009^{*}$ \\
\hline Communication & 14 & 51.9 & 9 & 33.3 & 1.89 & 0.17 \\
\hline Technology & 23 & 85.2 & 4 & 14.8 & 26.74 & $<0.001^{*}$ \\
\hline Customer satisfaction & 12 & 44.4 & 8 & 29.6 & 1.27 & 0.26 \\
\hline \multicolumn{7}{|l|}{ Total organizational climate: } \\
\hline Adequate & 13 & 48.1 & 0 & 0.0 & & \\
\hline Inadequate & 14 & 51.9 & 27 & 100.0 & 17.12 & $<0.001^{*}$ \\
\hline
\end{tabular}

(*) Statistically significant at $\mathrm{p}<0.05$

This table evidenced that, there was high significant difference between accredited and non-accredited regarding organizational design, co-work relations, culture/work environment, customer satisfaction and work process.

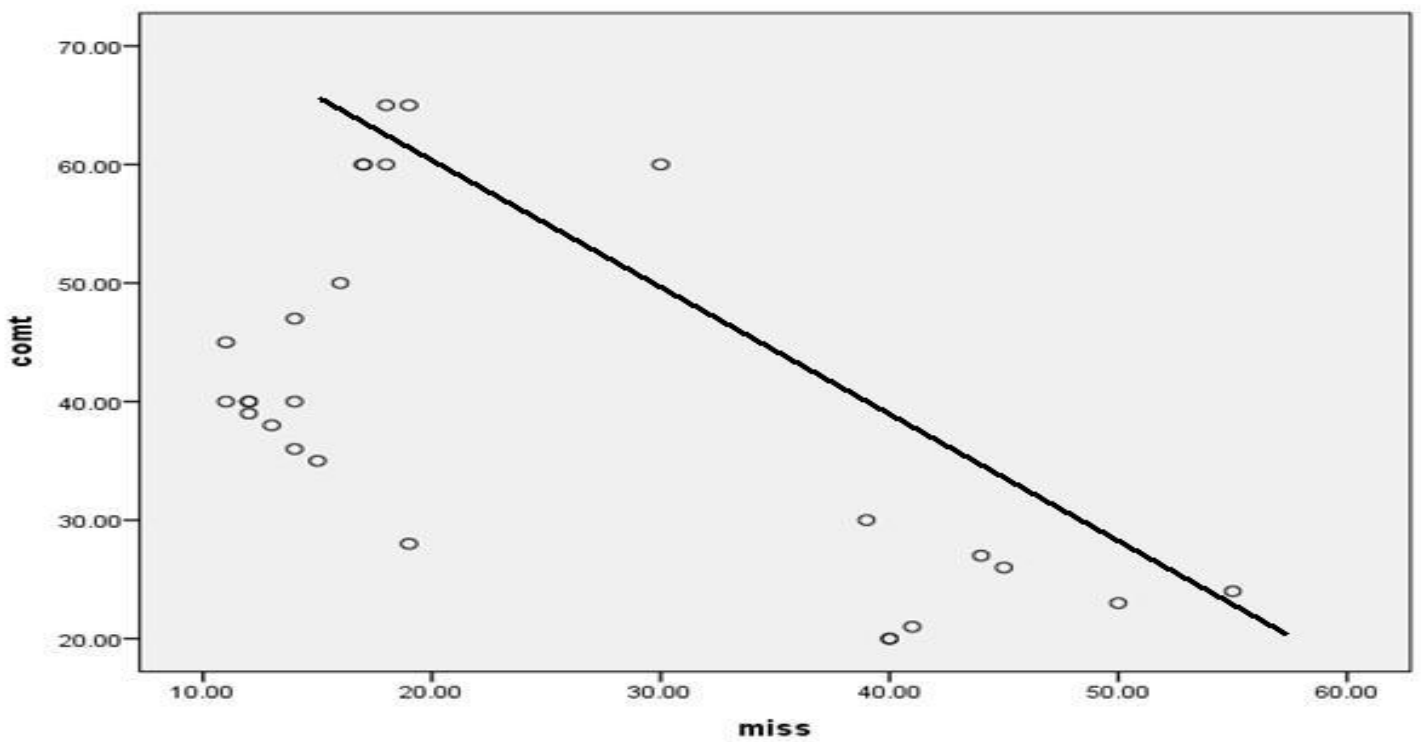

Fig (1): Correlation between the frequency of missing errors in prescribing and the score of organizational commitment $\mathrm{r}-.603 \mathrm{p}=0.017 \mathrm{P}<0.05$ negative correlation

This figure shows that there is negative correlation between organizational climate and frequency of errors.

\section{DISCUSSION}

The present study was conducted to evaluate the conformity of the prescription writing regarding the omission error to the standard of the MOHP, according to these errors, an intervention program was implemented and evaluated. 
Prescription writing is an important aspect in the healthcare system. Rational medication prescribing is closely associated with patient safety and good quality of life together with reasonable cost-effectiveness ${ }^{(5)}$.

In reviewing the literatures ${ }^{(6)}$ it was found that in most published studies of prescribing errors there are two main causes; the first is omission error and the second is the commission error. The main goal in this study is to focus on intervention that is essential, practical and can provide a conceptual framework for reducing the omission errors to the minimal possible degree.

After performing all the stages of the study from collecting personal questionnaire, determining the errors, to the face to face intervention training program according to the gap analysis and Pareto chart. The answer of this question proved to be that the educational intervention can decrease the omission error significantly.

In this study, there was no significant difference regarding Qualification of the physician and Experience working years between accredited and non-accredited units, which make the two groups homogenous in the comparison.

In all stages of the present study the accredited units had showed errors less frequent than that of the non-accredited units especially in the items of dose and route of the drug. The $\mathrm{P}$ value was $<0.05$ in items of dose of the drug, route of its administration, concentration of the drug, duration of its intake and the signature of the prescriber. This shows the effect of accreditation in the performance of these units and with continuous education and supervision the difference can decline.

The above findings were in agreement with Gadallah et al. ${ }^{(7)}$ who conduct a study on four reformed PHC units were compared with four nonreformed units in the Alexandria governorate regarding patients' and providers' satisfactions. Assessment of the satisfaction was done using survey questionnaire and focus group discussion. The provider survey questionnaire was carried out on all providers working in the selected units. Focus group discussion was done in one accredited health unit and another non- accredited unit. They showed that providers in the accredited PHCs were more satisfied than providers in non- accredited PHCs regarding availability of equipment, job satisfaction and income satisfaction. No significant differences were noticed between both groups regarding social relations with either colleagues or directors. The patient satisfaction was higher in accredited family health units compared to non-accredited units in all aspects: cleanness, doctors and nurses, waiting area and waiting time. During the focus group discussion, patients in the accredited PHCs expressed their satisfaction regarding the cleanness, privacy, attitude of doctors and nurse as well the waiting area and waiting time.

These prove that the accreditation gave the worker in the unit the feeling of responsibility and more care to be better.

It was notice that there were no errors in the name, the age of the patient and the date on contrast, the follow up is missed in all prescriptions, which was justified that the patient doesn't take a copy from the prescription as it is taken in the pharmacist. Regarding other items, it was found that the patient sex was missed in $94.4 \%$ of the prescriptions, the address was missed in $88.9 \%$ of the prescriptions, the diagnosis written in Arabic was missed in $44.4 \%$ of the prescriptions, the scientific name of the drug in Arabic was missed in $63 \%$ of the prescriptions, the concentration of the drug was missed in $61.1 \%$ of the prescriptions, the dose of the drug was missed in $63 \%$ of the prescriptions, the route of administration of the drug was missed in $31.5 \%$ of the prescriptions, the duration of the drug intake was missed in $68.5 \%$ of the prescription and the signature of the physician was missed in $75.9 \%$ of the prescriptions.

The prescriber's name, address and signature were on $83.3 \%, 9.6 \%$ and $81.9 \%$ of prescriptions respectively. The patient's name, age and sex were on $94.6 \%, 77.3 \%$ and $51.3 \%$. No prescription contained the patient's address and weight. Generic drug names were used in only $15.1 \%$ and strength of medication and dose units were included in $26.6 \%$ and $55.6 \%$ of prescriptions. Most prescriptions $(94.0 \%)$ had no quantity indicated and had only partial instructions for patient use $(90.7 \%)$; the diagnosis was included in about two-thirds ${ }^{(12)}$.

Ansari and Neupane ${ }^{(3)}$ found no error regarding the name, age, sex and address of the patients. The error in prescriptions regarding the prescriber's name, qualification, NMC registration number and signature were $85.4 \%, 99.6 \%, 99.6 \%$ and $15.7 \%$ respectively. Similarly, the symbol Rx was missing in $66.8 \%$. Dosage form, quantity, dose, frequency and route of administration were not mentioned in $12 \%, 60 \%, 19 \%, 10 \%$ and $63 \%$ of the prescriptions respectively. This after conduction of a cross sectional descriptive retrospective study was 
conducted at Nobel Medical Teaching Hospital, Biratnagar, Nepal during a time period from 15th November 2008 to 14th February 2009. A random sample of 268 prescriptions of patients written during a period of one year (18/06/2007 to $17 / 06 / 2008$ ) for ten different medical outpatient departments of the Hospital were reviewed and the analysis was carried out for determining the different types of errors in writing a prescription.

Calligaris et al. ${ }^{(\mathbf{8})}$ point prevalence survey, carried out in May 26-30 2008, involved 41 inpatient Units. Every parenteral or oral antibiotic prescription was analyzed for legibility (generic or brand drug name, dose, frequency of administration) and completeness (generic or brand name, dose, frequency of administration, route of administration, date of prescription and signature of the prescriber). Eight doctors (residents in Hygiene and Preventive Medicine) and two pharmacists performed the survey by reviewing the clinical records of medical, surgical or intensive care section inpatients. The results showed that the legibility was incompliance with $78.9 \%$ of generic or brand names, $69.4 \%$ of doses, $80.1 \%$ of frequency of administration, whereas completeness was fulfilled for $95.6 \%$ of generic or brand names, $76.7 \%$ of doses, $83.6 \%$ of frequency of administration, $87 \%$ of routes of administration, $43.9 \%$ of dates of prescription and $33.3 \%$ of physician's signature. Overall $23.9 \%$ of prescriptions were illegible and $29.9 \%$ of prescriptions were incomplete. Legibility and completeness are higher in unusual drugs prescriptions.

These differences in the results were due to the difference in sample size, type of study and different sitting.

The organizational climate is an important factor in quality of any service, so it has great effect in improving the errors. In this study the majority of physicians reported adequacy in the items of organizational design, senior management and work process, where other items were inadequate. The organizational climate is an item that need the support of the system of the ministry, thus it need a lot of work to be improved. Its improvement will give the worker in the institute push to advance their work. So this point is an important point for further studies.

Linzer et al. ${ }^{(9)}$ had conducted longitudinal study of physicians and patients in New York, Chicago, and the state of Wisconsin, including
Milwaukee and Madison. Physician surveys assessed office environment and organizational climate (OC). Stress was measured using a 4-item scale, past errors were self-reported, and the likelihood of future errors was self-assessed using the OSPRE (Occupational Stress and Preventable Error) measure. Among 420 physician respondents, predominantly from general medicine and family medicine practices, 38 percent described their office environment as busy, tending toward chaotic, while another 10 percent described their office environment as hectic or chaotic. Sixty-one percent agreed their work was stressful; 27 percent noted burnout symptoms; and 31 percent of respondents said they were at least moderately likely to leave their jobs within 2 years.

There was a significant difference in the score of the organizational climate between accredited and non-accredited unit in items of organizational design, co-work relations, culture/work environment, customer satisfaction and work process with $\mathrm{P}$ value $<0.001,<0.001$, $<0.001,0.009$ and $<0.001$ respectively. The accredited units had better score than nonaccredited and this supports the effects of accreditation.

This is supported by Al Tehewy et al. ${ }^{(\mathbf{1 0})}$ who found that the accreditation of the nongovernmental health units has a positive effect regarding patient satisfaction and the continuation of performance according to the accreditation standards compared with non-accredited health units, after conducted a study on sixty nongovernmental health units were selected as follows: 30 units already submitted for accreditation in three governorates and 30 pair-matched units not programmed for accreditation.

The above finding was also supported by Sierpińska and Ksykiewicz ${ }^{(11)}$, who conducted a study to discover whether there are any differences in the evaluation of organizational climate among the staff of hospital wards in hospitals with and without the Quality Certificate. The study covered 161 physicians and 339 nurses from 4 hospitals with accreditation and 17 units without the Quality Certificate. The study was carried out by the method of a diagnostic survey; the technique was a questionnaire form. The results of the study showed that physicians and nurses from hospitals with accreditation significantly more often evaluated the organizational climate in their wards in positive terms. The staff from hospitals without the Quality 
Certificate evaluated the atmosphere in their wards in more negative terms.

In the present study, it was found that there is negative correlation between organizational climate and frequency of prescription error. As the score of the organizational climate increases, the frequency of errors decreases.

This is in agreement with Linzer et al. ${ }^{(\mathbf{1})}$, who found that the higher score of the organizational climate, the less errors done.

It was noticed that the follow up item is not included in any prescription as it is already not taken by the patient, so the recall of the time of the follow up can be forgotten by the patient, which will lead to incomplete treatment.

In the present study all the physician, pharmacist and employee cooperated due to the presence of one of the accreditation supervisor with the researcher, so this overcome the problem of lack of cooperation which may be found in other researches. But some of the physicians were resistant as the thought that is time consuming and it doesn't affect the patient.

The study took more time than expected due to the absence of some physician, as a result the training program was postponed as the intervention was face to face and this took more time. This also was due to the lack of patients in some days that lead to waiting more time to collect the prescriptions.

The presence of the department of the accreditation support has another view that the physicians and pharmacists was consider the researcher as a part of the accreditation supervision and that take time and effort to show that was wrong for fear of the inaccuracy of data.

The reduction in prescribing errors can be achieved through proper training, continuous supervision and suitable accountancy.

Generalization of the results of this study can be extended to Zagazig city.

\section{CONCLUSION}

In the present study after assessing the prescriptions and then comparing with the standards of MOHP, it is evident that prescription errors are abundant and often occult. The handwritten prescriptions are associated with relatively higher error rates associated with prescription writing in the Zagazig city in all areas like doctors' signature, patient details and drug details and diagnosis of the disease.
With comparing the errors before and after the intervention program, it was proven that the intervention decreased the errors in both accredited and non-accredited units. The accredited units show less errors and higher score in the organizational climate than the non-accredited units.

Errors were predominately related to: the organizational structure of the prescribing process; the knowledge characteristics of the doctors; the communication patterns they used; the underlying assumptions they made about prescribing; and the unit work environment itself. Consideration of these factors may be important for the design and implementation of strategies to improve the quality and also educational programs in prescribing for junior doctors.

The prescriptions need more effort and support from all the related to it; from the doctors, senior managers to the accreditation committee and ministry of health.

\section{RECOMMENDATION}

The health care quality system should be improved for more patient safety.

Focusing on the patient safety and prescription writing in the curriculum of the medical students.

The health care services should fulfill the customers' anticipation for the seek of the patient satisfaction.

The health care providers should follow the guidelines of family medicine and the standard of accreditation.

\section{REFERENCES}

1. Miasso AI, Oliveira RC, Silva AE, Lyra DP, Gimenes FR, Fakih FT, Cassiani SH (2009): Prescription errors in Brazilian hospitals: a multi-centre exploratory survey Cad. SaúdePública, Rio de Janeiro, 25(2):313320.

2. Kuan M, Chua S and Mohmed R (2002): Noncompliance with prescription writing requirements and prescribing errors in an Outpatient Department. Malaysian Journal of Pharmacy. 1 (2): 45-50.

3. Ansari M and Neupane D (2009): Study on determination of errors in prescription writing: A semi-electronic perspective. Kathmandu Univ Med J (KUMJ). Jul-Sep; 7(27):238-41.

4. World Health Organization (2008): Summary of the evidence on patient safety.

5. Walley T, Mrazek M, Mossialos E (2005): Regulating Pharmaceutical Markets: improving efficiency and controlling costs in the UK. International Journal of Health Planning \& Management 20: 375-98. 
6. Carpenter L (2011): Error prevention tips. Available at:

CEdrugstorenews.com/userapp//lessons/lesson_view_ ui.cfm?lessonuid=401-000-11-102-H03. Accessed March 21, 2011.

7. Gadallah MA, Allam MF, Ahmed AMA (2009): Are patients and healthcare providers satisfied with health sector reform implemented in family health centres? QualSaf Health Care. doi:10.1136/qshc.2007.024364 1 of 5.

8. Calligaris L, Panzera A, Arnoldo L, Londero C, Quattrin R, Troncon M, Brusaferro S (2009): Errors and omissions in hospital prescriptions: a survey of prescription writing in a hospital Published: 13 May BMC Clinical Pharmacology, 9:9 doi: 10.1186/14726904-9-9.

9. Linzer M, Manwell L, Mundt M, Williams E, Maguire A, McMurray J, Beth Plane M (2005): Organizational Climate, Stress, and Error in Primary
Care: The MEMO Study Advances in Patient Safety: Vol. 1.

10. Al Tehewy M., Salem B., Habil I. AND El Okda S. (2009): Evaluation of accreditation program in nongovernmental organizations' health units in Egypt: short-term outcomes International Journal for Quality in Health Care; Volume 21, Number 3: pp. 183-189.

11. Sierpińska L and Ksykiewicz A (2003): Organizational climate as a precondition of effective work of a therapeutic team. Ann UnivMariae Curie Sklodowska Med.; 58(2):136-41.

12. Linzer M, Manwell L, Mundt M, Williams E, Maguire A, McMurray J, Beth Plane M (2005): Organizational Climate, Stress, and Error in Primary Care: The MEMO Study Advances in Patient Safety: Vol. 1. 


\section{أخطاء الوصفات الطبية بوحدات طب الأسرة}

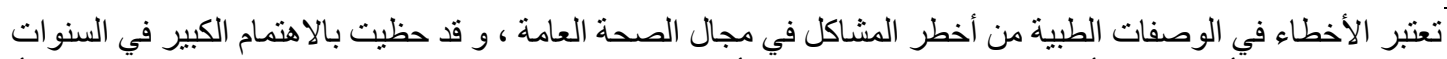

المقدمة :

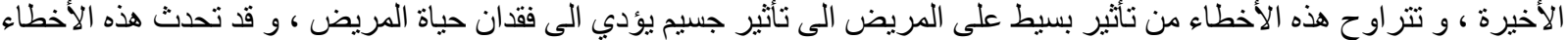

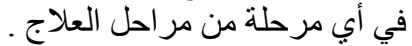

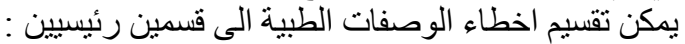

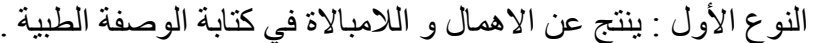

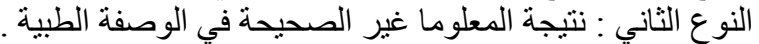
و في سنة 2005 أظهرت الدر اسات أن الأخطاء الطبية كانت السبب في وفاة أكثر من 7 الاف شخص في السنة و وفاة 6,5 من كل مائة

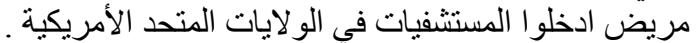

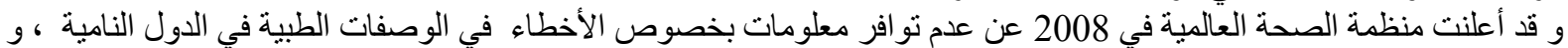
لذللك نحتاج الى وجود قاعدة بيانات في هذه الدول بخصوص الدي الوصفات الطبية بها . 